\title{
USOS DE LOS MEDIOS DE TRANSPORTE Y ACCESIBILIDAD URBANA. UN ESTUDIO DE CASO EN EL ÁREA METROPOLITANA DE GUADALAJARA, MÉXICO
}

\author{
Fernando Calonge Reillo ${ }^{l}$ \\ Centro Universitario de Tonalá- Universidad de Guadalajara, México.
}

\section{RESUMEN}

En las últimas dos décadas la organización del territorio y la disposición de los sistemas de transportes ha condicionado seriamente la forma como los hogares segregados de bajos recursos acceden a los principales servicios urbanos y lugares para el desarrollo de sus actividades más básicas. Esta problemática ha llevado al desarrollo de los estudios sobre la accesibilidad urbana. No obstante, la mayor parte de las investigaciones se han realizado en el contexto de las urbes desarrolladas. Este artículo contribuye a afianzar las investigaciones que se ubican en el contexto de los países pobres o en desarrollo, y tiene la intención de mostrar cómo las poblaciones de espacios no centrales y periféricos de la metrópolis llegan y se desplazan a sus centros de trabajo y educativos, a los comercios, clínicas y hospitales. Se presentan los principales resultados de una encuesta de movilidad realizada en el Área Metropolitana de Guadalajara, México en 2015, con la intención de matizar y complementar los principales hallazgos y recomendaciones sobre la accesibilidad urbana que se acuñan desde los países desarrollados.

Palabras clave: Accesibilidad urbana; países en desarrollo; periferias urbanas; servicios urbanos; transporte.

\section{TRANSPORTATION MODE CHOICE AND URBAN ACCESSIBILITY. A CASE STUDY IN GUADALAJARA METROPOLITAN AREA, MEXICO}

\section{ABSTRACT}

In the last twenty years, the structuring of the urban territory and the organization of the systems of transportation have influenced how poor and segregated households access different locations where urban services and main urban activities are accommodated. These situation shave motivated academics to study urban accessibility, especially in developed metropolitan regions. This paper comes to grips with similar phenomena in the context of a developing urban area trying to extend and nuance the existing body of knowledge. The paper reflects in how populations in non-central and peripheral areas travel to their job, educational and shopping centers, and to clinics and hospitals. It is based on a survey carried in 2015 in Guadalajara Metropolitan Area, México, and resumes the main outcomes to update the proposals that are framed in the developed countries.

Keywords: Urban accessibility; developing countries; urban peripheries; urban services; transportation.

\footnotetext{
${ }^{1}$ Departamento de Ingenierías, Centro Universitario de Tonalá, Universidad de Guadalajara, Av. Nuevo Periférico No. 555 Ejido San José Tatepozco, C.P. 45425, Tonalá Jalisco, México. Correo electrónico: fercalonge@yahoo.es
}

Fecha de recepción: 12 de abril de 2016. Fecha de aceptación: 14 de mayo de 2016. 


\section{INTRODUCCIÓN}

En las últimas décadas la realidad urbana de los países en desarrollo ha experimentado una serie de cambios que hace más compleja la forma en que las poblaciones viven y aprovechan sus entornos. El gran crecimiento poblacional territorial de las ciudades, y las dificultades por articular los distintos espacios urbanos, han hecho que las poblaciones más desfavorecidas encuentren cada vez más problemas para poder acceder a los servicios básicos, o para poder aprovechar las fuentes de trabajo. Todos estos problemas han sido reunidos y analizados con profundidad desde los estudios sobre la accesibilidad urbana.

Buena parte de las investigaciones sobre la accesibilidad se han ubicado en el contexto de los países desarrollados: Estados Unidos, Canadá, Australia, la Unión Europea o Japón. En estos entornos, estos problemas, en ocasiones, se han vinculado con la apuesta por recuperar la ciudad compacta, la multifuncionalidad espacial y los medios de transporte no motorizados, complementados con los colectivos que permitan aprovechar la riqueza del entorno más inmediato. Por suerte existe también un buen número de estudios que se han centrado en los países en desarrollo, ya sea el África Subsahariana, Oriente Próximo o la región Latinoamericana. Estos estudios muestran realidades muy distintas a las que se encuentran en los países desarrollados, y aconsejan repensar las fórmulas para mejorar la accesibilidad de las poblaciones, sobre todo de aquellos grupos marginados ubicados en las periferias urbanas. El propósito de este artículo es ayudar a incrementar esta base empírica, especificando en concreto cómo el uso diferenciado de los medios de transporte en municipios no centrales de una conurbación, incide en los diferentes niveles de accesibilidad urbana. Para ello, se realizó una encuesta en el año 2015 en los municipios del sur del Área Metropolitana de Guadalajara, México (Tlaquepaque, Tonalá, Tlajomulco y El Salto).

El presente artículo está estructurado en una primera parte de naturaleza teórica que se orienta a establecer un marco conceptual para la consideración de la accesibilidad urbana, a detectar sus principales condicionantes y a especificar cómo se produce la accesibilidad a las principales actividades necesarias para los hogares: trabajo, educación, sanidad y compras. En segundo lugar, se establece la metodología y el alcance que tiene este artículo. Acto seguido se presentan los principales resultados de la encuesta aplicada, caracterizando la accesibilidad de que gozan los distintos grupos de hogares en función de cómo realizan su gasto en transporte y de los medios de transporte que emplean.

\section{LA ACCESIBILIDAD URBANA}

\subsection{LA OPERACIONALIZACIÓN DE LA ACCESIBILIDAD}

Durante los dos últimos decenios, una profusa discusión teórica ha servido para dimensionar y asentar una concepción más o menos canónica de la accesibilidad. El enfoque de la accesibilidad intenta descubrir las condiciones para que las poblaciones materialicen las distintas oportunidades espaciales a través del sistema de transporte (HULL, 2011; HOERNER et al., 2015). Ese estudio de la accesibilidad, ha quedado complementado con la integración de tres elementos principales.

En primer lugar está el estudio sobre los sistemas de transporte y la manera cómo condicionan que unos lugares estén mejor o peor conectados con otros lugares en función de su integración con la red (OHNMACHT et al., 2009). Este primer elemento se complementa con la consideración de un segundo elemento: la propia estructura del territorio y la distribución de los diferentes lugares donde se obtienen beneficios y oportunidades para el desarrollo de actividades fundamentales (ADEEL et al., 2016; NEUTENS, 2015). Con estos dos elementos, el concepto de accesibilidad se refiere a la manera cómo el sistema de transporte articula las diferentes localizaciones donde se desarrollan los principales servicios y actividades dentro de la estructura territorial (CAHILL, 2010).

Posteriormente se añadió un tercer espacio de reflexión y de análisis que complementa la dimensión excesivamente geográfica que se estaba alcanzando. Así, se advirtió que los bienes y servicios son accesibles a través de los sistemas de transporte, pero para un público 
USOS DE LOS MEDIOS DE TRANSPORTE...

(KELLERMAN, 2006) o unos sujetos particulares (HERNÁNDEZ, 2012; LAU, 2011). En esa medida, la accesibilidad ahora sería un concepto bisagra que comunicaría la estructura del territorio, el sistema de transportes y las poblaciones urbanas con sus correspondientes necesidades (BRINDLE, 2003).

Ha sido también muy debatido en qué podrían consistir estas necesidades. La literatura ha fluctuado entre designar un término medio definido según una concepción más o menos establecida de las necesidades domésticas (LAU et al., 2003) o de las necesidades socialmente preponderantes (LUCAS, 2004). Otros autores, por el contrario, han propuesto reparar en la autonomía que puedan tener los ciudadanos (MANDERSCHEID, 2009) para poder considerar las actividades que en un determinado momento de su ciclo vital son significativas para ellos (LE VINE et al., 2013), por encima de las definiciones del investigador o de aquellas socialmente estandarizadas.

Al ser el concepto de la accesibilidad intrínsecamente multidimensional, se ha sugerido la necesidad de emplearlo meticulosamente, para evitar que la obtención de resultados divergentes sea debida a la utilización de metodologías disímiles (WIDENER et al., 2013). En esa medida, las propuestas de realización son concordantes con las dimensiones anteriormente analizadas. Un grupo de autores aboga por traducir la accesibilidad a una cuestión de medición de las distancias, ya sea a las residencias, los centros de trabajo o las paradas de transporte público (SEO et al., 2013). Algunas matizaciones que siguen dentro de esta propuesta consisten en considerar las distancias que separan a los sujetos de los lugares relevantes, pero a lo largo de todos sus desplazamientos diarios (WIDENER et al., 2013), o en apuntar que esas distancias no pueden ser las que describan líneas rectas de los sujetos a los lugares, sino venir particularizadas por las rutas y vialidades efectivamente tomadas (BLANFORD et al., 2012).

Otras propuestas intentan aproximarse a los sujetos y usuarios basándose en los tiempos de traslado que les toma el llegar a esos lugares significativos (FARBER et al., 2011; WIEDENER et al., 2015), ya sea en automóvil (HORNER et al., 2015) o en otros medios de transporte. Esta perspectiva espacio-temporal, permite hacer más complejos los modelos, de manera que se integren los tiempos de desplazamiento en las temporalidades propias de las organizaciones domésticas o laborales de las que se depende (FARBER, et al., 2014: 150), o en las rutinas temporales de los propios sujetos y hogares (NEUTENS, 2015; HERNÁNDEZ et al., 2015). Este cambio de perspectiva, lleva a acercamientos más realistas a las condiciones de accesibilidad particulares, en la medida en que permite considerar, por ejemplo, las estrategias temporales de los hogares dentro de entornos complejos y caracterizados por incertidumbres tales los fluctuantes horarios en la operación del transporte público o por las variadas condiciones del tráfico (SWEET et al., 2011; ETTEMA et al., 2007)

Por último, hay autores que han profundizado en este énfasis sobre las estrategias de accesibilidad aplicando complejos modelos de toma de decisiones y de maximización de utilidades (SALZE et al., 2011). Así, se propone contemplar cómo los sujetos acometen ciertas estrategias para maximizar sus utilidades totales, considerando las diversas posibilidades de encadenar desplazamientos o actividades diversas, o de integrar estos viajes junto al resto de tareas personales (DONG et al., 2006). Los mayores niveles de accesibilidad implicarían una maximización de las utilidades obtenidas en la toma de decisiones.

\subsection{ALGUNOS CONDICIONANTES PARA LA ACCESIBILIDAD URBANA}

En los últimos decenios la accesibilidad urbana se ha visto comprometida por una gran dispersión en el territorio de residencias, centros educativos y laborales, hospitales o centros comerciales. La gran dispersión urbana que se evidencia en buena parte de las ciudades, sobre todo en los países en desarrollo, ha sido posibilitada por una extensión en el uso del automóvil. El automóvil ha permitido que viviendas, comercios, trabajos y servicios urbanos se ubiquen dispersamente, y los hogares puedan desplazarse flexiblemente de un lugar a otro según sus necesidades (CAHILL, 2010; URRY, 2006). En particular, se establece un círculo vicioso por el cual un territorio disperso fuerza un mayor uso del automóvil que, a su vez, permite desconcentrar aún más esos espacios significativos (KIM et al., 2008). 
En esta nueva estructura territorial, el disponer del automóvil puede articularse en un nuevo eje de estratificación debido a la forma como condiciona las accesibilidades urbanas. No en vano existen estudios que demuestran que la adquisición de un automóvil dentro de los hogares es un medio seguro de ampliar el número de trabajos a los que se puede aspirar (WANG et al., 2015), o un medio que permite aumentar los ingresos mensuales obtenidos (SERULLE et al., 2016). Al mismo tiempo, y bajo una lógica del mercado, son las poblaciones pobres las que menos capacidad tienen a la hora de decidir el lugar de su residencia (PINJARI et al., 2011), y por tanto son expulsadas a los espacios más lejanos y periféricos de las urbes, sobre todo en los países en desarrollo (JIRÓN, 2004). Sin el acceso al automóvil, y en un contexto de periferia, las poblaciones pobres tienen más dificultades de acceder a los trabajos, el comercio o los servicios urbanos (SABATINI et al., 2008) y, por tanto, habrían de incurrir en mayores costos temporales (LAU et al., 2003) y monetarios (ZOLNIK, 2012).

Es importante detenerse en esas condiciones del hábitat en los entornos urbanos no centrales por cuando en ellos es donde se ubica nuestra investigación. La principal característica de buena parte de los territorios periféricos es la ausencia de los servicios más básicos necesarios para la reproducción de los hogares: agua, electricidad, salud, educación, comercio o transporte (LINDÓN VILLORIA, 1997: 9; SOCIAL EXCLUSION UNIT, 2003; EIBENSCHUTZ et al., 2011; GARLAND et al., 2007). A la lejanía de estos servicios, hay que sumar la existencia de una estructura vial y carretera que por insegura, mala conservación y trazo, o escasa permeabilidad, aleja todavía más a las poblaciones segregadas (BEJLERI, 2011; HAOULUAN et al., 2016; DELGADO et al., 2011).

Las características del sistema de transporte en los espacios de periferia tampoco ayudan. En específico, se ha documentado una menor disposición de automóviles, la carencia de infraestructura suficiente para la movilidad no motorizada, una menor cobertura de los servicios de transporte formal, y la aparición de operadores informales que establecen sus rutas preferentemente a lo largo de las principales, pero escasas carreteras que conectan con los centros urbanos (DIAZ OLVERA et al., 2013; SRINIVASAN et al., 2005). Es el transporte colectivo de autobuses y minibuses el que presta un servicio menos eficiente a las poblaciones periféricas, forzándoles a realizar trayectos más complejos y lentos (LAU, 2011: 235) Las inconveniencias se sienten especialmente los fines de semana, en franjas temporales cuando hay menos servicio del transporte colectivo (COVENEY et al., 2009; FARBER et al., 2014), cuando es más imprevisible el cumplimiento de los horarios (ETTEMA et al., 2005), o cuando se necesita ir de una zona periférica de la ciudad a otra igualmente periférica (LAU, 2011). Estos pobres niveles de servicio que ofrece el transporte colectivo en las periferias hace que en muchas ocasiones la población contemple evitarlo (ADEEL, et al., 2016) resignándose a desarrollar el grueso de sus actividades en esa proximidad empobrecida de la periferia.

Otra de las variables que incide en la accesibilidad urbana es que el precio del transporte colectivo sea costeable para todos los hogares (MOAVENZADEH et al., 2007). En esa dirección toda una serie de estudios han intentado computar el porcentaje de los ingresos familiares que se destinan a los gastos en transporte. Esa proporción es muy variable y depende de las características del área metropolitana, de la ubicación de la residencia y de los ingresos del hogar; así, se han consignado gastos que van del $12 \%$ en una ciudad con elevadas densidades de población como Singapur (LAU, 2011), hasta el 20`9\% en otra ciudad como Houston mucho más dispersa (YANG et al., 2006). Estos gastos tienden a aumentar cuanto más periférica es la ubicación del hogar (ZOLNIK, 2012).

El que los hogares más pobres gasten una más elevada proporción en transporte (DIAZ OLVERA et al., 2013) condiciona que sus miembros tengan que acceder a medios de transporte más lentos (LAU, 2011), en especial los motorizados (PLAUT, 2004; SRINIVASAN et al., 2005), y que muestren una tendencia a vivir en entornos urbanos compactos donde los servicios se encuentren próximos (LEWIS et al., 2010). Todo ello concurre en que para poder acceder a los servicios más básicos, las poblaciones marginadas de las periferias urbanas se vean obligadas a realizar traslados más largos (MASOUMI, 2014; DIAZ OLVERA et al., 2013), y en general, sin apenas opciones de locación y desplazamientos, se encuentran con serias dificultades para planificar viables estrategias de movilidad por la ciudad (SUSILO et al., 2014). 
Frente a esta situación, se ha sugerido que una mejora en la densidad de la cobertura del transporte en las áreas periféricas puede ampliar las opciones que contemplar en la formación de estrategias de movilidad (SUSILO et al., 2014) sobre todo cuando encontramos considerables niveles de densidad poblacional (CASTILLO-MANZANO et al., 2009). Sin embargo, la dominante de las periferias urbanas no es esa; ante las bajas densidades generalizadas, el transporte público tiene serias limitaciones para prestar cobertura a la población (HORNER et al., 2015) y más si a la baja densidad le añadimos las condiciones de amplia dispersión de servicios en estas zonas limítrofes (DIAZ OLVERA et al., 2013).

Otra serie de propuestas se centran en el desarrollo del territorio. Así, se ha señalado (BANISTER et al., 1995) que la estructura del territorio y la ubicación de los servicios no responde necesariamente de una manera causal a las innovaciones en los sistemas de transporte, y que es necesario integrar análisis más complejos que valoren el papel que la propia planificación del territorio puede tener. De esta forma se ha indicado cómo es conveniente planificar más certeramente la ubicación de los puestos de trabajo o de las áreas residenciales (BANISTER, 2002) para mejorar las condiciones de accesibilidad de las poblaciones. Una primera vía para materializar este tipo de preocupaciones lo componen los modelos 'Land Use Transport Interaction' (LUTI) que, integrando las tomas de decisiones locacionales de residentes (demandantes) y de productores de bienes y servicios urbanos (ofertantes) bajo la lógica de un análisis de mercado en diferentes subsistemas, intentan explicar y predecir la ubicación territorial de residencias, empleos, comercios o centros educativos (SIMMONDS et al., 1999; WEGENER, 2008; BATTY, 2009). Otra aproximación que enfatiza la dimensión de planificación del territorio para superar los obstáculos a la accesibilidad son los estudios sobre el desarrollo orientado al transporte, que implica el desarrollo de centros urbanos alrededor de grandes intercambiadores del transporte, y caracterizados por modalidades de elevadas densidades poblacionales y usos mixtos de suelo (NEWMAN, 2009).

\subsection{LA ACCESIBILIDAD A LOS PRINCIPALES SERVICIOS URBANOS}

No hay espacio en este apartado para discutir en profundidad los aspectos del acceso de los hogares y sujetos a cada una de las actividades básicas de los hogares. Sin embargo, se ofrecerán las principales tendencias encontradas en la literatura sobre el acceso al comercio, a la educación, la salud y los centros de trabajo que son los emplazamientos que se contemplarán en el estudio empírico.

En primer lugar tenemos el caso de las compras, que son las que suscitan un mayor número de viajes, especialmente de proximidad (MARQUET et al., 2014). El acceso a este tipo de servicios ha estado condicionado por una creciente concentración del comercio (CLIFTON et al., 2004), que se organiza bajo el formato de grandes superficies (BIBA et al., 2006) a las que es casi imprescindible llegar en automóvil (REIMERS, 2013). Esto, unido a que en el ramo de la alimentación tienden a desaparecer los comercios de perecederos a favor de los de comida rápida (HEYNEN, 2006), ha motivado que en el entorno urbano aparezcan los llamados 'desiertos alimentarios', es decir, unidades geográficas que carecen de supermercados y comercios que provean de alimentos nutritivos (HORNER et al., 2014). Estas circunstancias condicionan que los integrantes de los hogares pobres sufran peores condiciones de acceso para realizar sus compras cotidianas, al tener que realizar un mayor número de salidas caminando, a comercios cercanos y que ofrecen peores precios y calidades (FARBER et al., 2011; COVENEY et al., 2009; DIAZ OLVERA et al., 2003).

En lo que respecta al acceso a los servicios educativos, buena parte de la literatura se centra en el estudio de los medios de transporte utilizados, y en la conveniencia de favorecer el transporte no motorizado como una forma de desarrollar hábitos saludables en los escolares (FAULKNER et al., 2010; MCDONALD et al., 2014). En ese sentido se ha estudiado los obstáculos que para la accesibilidad a la educación pueden imponer las distintas características del entorno, especialmente la existencia de carreteras y tráfico pesado en las inmediaciones de los planteles o las largas distancias existentes (BEJLERI et al., 2011; FITZPATRICK et al., 2012). Esta serie de obstáculos se acrecentarían según se sube de nivel educativo, al existir menos centros educativos y situados en mayor lejanía. En estos estadios, el tener que utilizar el 
transporte motorizado estaría penalizando a los grupos sociales de rentas más bajas que no tendrían los recursos suficientes para su transporte, comprometiendo su accesibilidad a la educación media y superior (DIAZ OLVERA et al., 2003; KENYON, 2011).

Sobre el acceso a los servicios sanitarios, se ha señalado que uno de los obstáculos más difíciles de superar es la distancia a los centros de salud, sobre todo en los países en desarrollo (AOUN et al., 2015; BLANDFORD et al., 2012), puesto que en ellos una buena proporción de los pacientes no tienen otro medio para desplazarse que ir caminando (DIAZ OLVERA et al., 2003). El largo tiempo que se debe empeñar para poder acudir a una cita médica, hace que sea más difícil compaginar este tipo de actividades dentro del horario cotidiano de los sujetos que, en el caso de aquellos más pobres, muestra mayores niveles de fijeza e inflexibilidad (HERNANDEZ et al., 2015). En particular, existen áreas de la atención sanitaria donde es más grave la falta de acceso de las poblaciones pobres, como son las consultas de pediatría y la salud infantil (YANG et al., 2006) y el acceso a los hospitales de especialidades (HORNER et al., 2015).

Finalmente la ausencia de correspondencia entre la ubicación de los puestos de empleo y las residencias de los trabajadores, especialmente de los menos cualificados, se consigna como uno de los principales impedimentos en el acceso a los trabajos (SERULLE et al., 2016). Esta no correspondencia es más acusada conforme más nos alejamos del centro y nos desplazamos hacia las zonas periféricas (WANG et al., 2015). En el caso de las rentas más bajas, la literatura considera una doble posibilidad. Por un lado las dificultades para desplazarse al trabajo pueden ocasionar que los trabajadores opten por empleos ubicados en sus inmediaciones, pero peor remunerados (RODRÍGUEZ VIGNOLI, 2008), a los que en ocasiones se desplazan por medios no motorizados (PLAUT, 2004). Por otro, los trabajadores pueden mantener su aspiración de buscar mejores opciones laborales en espacios distantes, para lo cual realizarán viajes más largos sobre todo en los autobuses o microbuses (LAU, 2011), y superando algunas dificultades como las restricciones de horarios cuando los turnos laborales son extemporáneos (LAU et al., 2003).

\section{METODOLOGÍA Y ALCANCE}

Como ha quedado de manifiesto en buena parte de la literatura revisada, las peores condiciones para la accesibilidad urbana se presentan en las periferias de las metrópolis y atañen a las poblaciones de menos recursos. La investigación que subyace a este artículo busca indagar por cómo el uso de los medios de transporte condiciona el acceso de las poblaciones a las principales actividades, en el contexto de los municipios no centrales del Área Metropolitana de Guadalajara, México. Aunque existen excepciones que han sido documentadas, no abundan todavía este tipo de estudios que se ubican en el contexto de los países pobres o en desarrollo. Con los resultados que se ofrecen en este artículo, que son de naturaleza exploratoria, busco cubrir parte de esta laguna, y ofrecer unas provisionales hipótesis para poder encauzar futuras investigaciones sobre la accesibilidad urbana en los países en desarrollo.

Él Ârea Metropolitana de Guadalajara está compuesta por los municipios de Guadalajara, Zapopan, Tlaquepaque, Tonalá, Tlajomulco y El Salto. Conjuntamente constituyen una conurbación de cerca de 4'7 millones de habitantes. Son los cuatro últimos municipios los que se conurbaron más tardíamente y, por tanto, los que ocupan los espacios no centrales de la metrópolis. Aparte de ser los municipios periféricos, sus poblaciones son las que disponen de menores recursos monetarios y de accesibilidad.

Este artículo está basado en la realización de una encuesta de movilidad en esos cuatro municipios del sur del Área Metropolitana de Guadalajara (AMG) que indagó aspectos centrales para los propósitos del argumento, como gastos en los medios de transporte, medios de transporte utilizados para acudir a las distintas actividades, tiempos de desplazamiento y otras variables sobre la conveniencia del transporte en función del tipo de actividad desarrollada. La encuesta se realizó en hogares, elegidos aleatoriamente, e integra 800 cuestionarios cumplimentados que permiten establecer conclusiones para el conjunto muestral con un margen de error de +/- 4'31\%. La encuesta fue llevada a cabo en el mes de mayo de 2015, y se ubica dentro del marco del proyecto de investigación: El sistema de transportes y la accesibilidad en 
los municipios del sur del Área Metropolitana de Guadalajara, que contó con la financiación de la convocatoria de Ciencia Básica del Consejo Nacional de Ciencia y Tecnología (CONACyT) de México en el año 2013.

TABLA 1

Ingresos per cápita mensuales y acceso al automóvil por municipios del AMG

\begin{tabular}{|l|r|r|}
\hline & $\begin{array}{r}\text { Media ingreso mensual per } \\
\text { cápita }\end{array}$ & $\begin{array}{r}\text { Porcentaje sujetos acceso a } \\
\text { automóvil }\end{array}$ \\
\hline Guadalajara & 3300 & $58,2 \%$ \\
\hline EI Salto & 2106 & $44,8 \%$ \\
\hline Tlajomulco & 3371 & $48,9 \%$ \\
\hline Tlaquepaque & 2724 & $51,3 \%$ \\
\hline Tonalá & 2544 & $49,2 \%$ \\
\hline Zapopan & 4011 & $62,6 \%$ \\
\hline Total & 3288 & $55,8 \%$ \\
\hline
\end{tabular}

Fuente: Explotación propia de Encuesta Intercensal 2015 INEGI

Para el análisis estadístico se ha procedido a realizar un análisis de conglomerados, que busca distribuir los casos de una muestra en distintos grupos según son los valores que representan cada uno de los hogares según ciertas variables estratégicas. Para realizarlo se ha recurrido al método Ward, uno de los de mayor uso por su capacidad de obtener mejores niveles de discriminación en las agrupaciones obtenidas, y que busca minimizar las distancias al cuadrado de cada uno de los casos en la composición de los distintos clústeres. Para calibrar las asociaciones entre variables, cuando estamos ante variables dependientes numéricas, se ha procedido a aplicar un modelo ANOVA de diferencias de medias, que busca determinar que la diferencia de medias es significativa, para más de dos subgrupos determinados por la variable independiente. Cuando se trata de variables nominales, la asociación se ha calibrado recurriendo a la V de Cramer, que, basándose en el coeficiente Phi, sin embargo sí permite que para tablas de más de 2 categorías el valor de la asociación no supere en ningún caso el 1. En estos casos la ausencia de asociación es representada por el valor 0 , y la asociación perfecta por el valor 1 .

\section{RESULTADOS Y DISCUSIÓN}

\subsection{EL GASTO EN TRANSPORTE Y EL USO DE LOS DISTINTOS MEDIOS}

Para comenzar, se procedió a establecer una tipología de hogares según la forma como estructuraban sus gastos en el transporte. Para hacerlo, se aplicó un análisis de conglomerados que integró las variables sobre los porcentajes del ingreso familiar que se destinaban a sufragar los gastos en: operación de automóvil, de motocicletas, de bicicletas, de uso del microbús, del transporte masivo, del taxi y del mototaxi. Se generaron tres grandes grupos de hogares en función de la estructura de su gasto, que se distribuyen de la siguiente manera:

El primer grupo es el de quienes tienen un menor gasto total en transporte (un 13,42\% de los ingresos familiares), y destinan este gasto a sufragar el costo del pasaje en el transporte colectivo preponderante, los microbuses. El segundo grupo es el de quienes realizan un considerable gasto en transporte (el $31,47 \%$ de los ingresos totales), y lo destinan fundamentalmente a la operación del automóvil. Finalmente, existe un tercer grupo con menor número de hogares representados, y para quienes la carga de los gastos en transporte es tan pesada que supone el $44,04 \%$ de sus ingresos, y los destinan preferentemente al microbús, aunque también al transporte masivo. 
TABLA 2

Grupos de hogares según la estructura porcentual de su gasto en transporte

\begin{tabular}{|c|c|c|c|}
\hline & $\begin{array}{r}\text { Grupo 1. Gasto } \\
\text { reducido en microbús. } \\
49 \text { '3\% de los casos }\end{array}$ & $\begin{array}{r}\text { Grupo 2. Amplio } \\
\text { gasto en automóvil. } \\
\mathbf{3 8} 5 \% \text { de los casos }\end{array}$ & $\begin{array}{r}\text { Grupo 3. Amplio } \\
\text { gasto en microbús. } \\
12{ }^{\prime} \% \text { de los } \\
\text { casos }\end{array}$ \\
\hline$\%$ coche & 0,81 & 22,33 & 4,31 \\
\hline$\%$ moto & 0,84 & 0,66 & 0,04 \\
\hline$\%$ bicicleta & 0,36 & 0,41 & 0,35 \\
\hline \% microbús & 7,83 & 5,89 & 31,39 \\
\hline $\begin{array}{l}\% \text { transporte } \\
\text { masivo }\end{array}$ & 0,69 & 0,90 & 5,69 \\
\hline$\%$ taxi & 1,49 & 0,93 & 1,40 \\
\hline$\%$ mototaxi & 1,40 & 0,35 & 0,85 \\
\hline$\%$ gasto total & 13,42 & 31,47 & 44,04 \\
\hline
\end{tabular}

Fuente: Encuesta de Movilidad Municipios Sur AMG 2015

Conforme se ha reconocido en la literatura, el gasto en transporte incide de una forma muy importante en el medio utilizado para desplazarse a realizar las diferentes actividades. A este respecto se han encontrado asociaciones significativas entre la variable grupo de hogares según estructura del gasto en transporte, y los medios de transporte utilizados para acudir a realizar las principales actividades. La asociación más fuerte se ha dado entre el grupo de hogares según estructura del gasto en transporte y los medios utilizados para ir al hospital (V de Cramer 0,436), y la asociación más débil entre esos mismos grupos de hogares y los medios de transporte utilizados para ir a la escuela (V de Cramer 0,261). Los porcentajes concretos se muestran en las siguientes tablas:

TABLA 3

Medios de transporte utilizados para acudir a realizar las diferentes actividades del grupo de hogares de gasto reducido en transporte

\begin{tabular}{|c|r|r|r|r|r|}
\hline & Compras & Escuela & Trabajo & Médico & Hospital \\
\hline $\begin{array}{c}\text { Andando o } \\
\text { bicicleta }\end{array}$ & $62,7 \%$ & $62,6 \%$ & $31,9 \%$ & $45,4 \%$ & $5,3 \%$ \\
\hline Automóvil & $12,3 \%$ & $6,6 \%$ & $10,5 \%$ & $9,8 \%$ & $10,0 \%$ \\
\hline $\begin{array}{c}\text { Transporte } \\
\text { público }\end{array}$ & $25,0 \%$ & $30,8 \%$ & $57,6 \%$ & $44,8 \%$ & $84,7 \%$ \\
\hline
\end{tabular}

Fuente: Encuesta de Movilidad Municipios Sur AMG 2015

TABLA 4

Medios de transporte utilizados para acudir a realizar las diferentes actividades del grupo de hogares de gasto considerable en automóvil

\begin{tabular}{|c|r|r|r|r|r|}
\hline & Compras & Escuela & Trabajo & Médico & Hospital \\
\hline $\begin{array}{c}\text { Andando o } \\
\text { bicicleta }\end{array}$ & $38,9 \%$ & $48,0 \%$ & $18,1 \%$ & $26,6 \%$ & $3,6 \%$ \\
\hline Automóvil & $55,4 \%$ & $28,9 \%$ & $58,6 \%$ & $51,1 \%$ & $69,6 \%$ \\
\hline $\begin{array}{c}\text { Transporte } \\
\text { público }\end{array}$ & $5,7 \%$ & $23,0 \%$ & $23,3 \%$ & $22,3 \%$ & $26,7 \%$ \\
\hline
\end{tabular}

Fuente: Encuesta de Movilidad Municipios Sur AMG 2015

Según se comprueba, los hogares que tienen un gasto reducido en transporte, intentan desplazarse por los medios no motorizados, que son los más económicos, para realizar aquellas actividades que en principio tendrían mayor disponibilidad de realizarlas en un entorno de 
proximidad: así sucede fundamentalmente con el acudir a realizar las compras o el acudir a la escuela, y en menor medida, recibir atención médica primaria. Para otras actividades donde se encontrarían más dificultades para localizarlas en la proximidad, este grupo opta fundamentalmente por emplear el transporte público: así sucede con el trabajo, donde el 57,6\% de los hogares utiliza este medio, y con las citas al hospital, donde el 84,7\% de los hogares se desplaza en transporte público.

Dado lo modesto del ingreso de buena parte de los hogares en los municipios no centrales del AMG, el uso del automóvil no es indiscriminado, ni siquiera para el grupo de hogares que realizan un gasto considerable en transporte orientado a este medio. Estos hogares utilizan el automóvil sobre todo para acudir al hospital (el 69,6\%), y en menor proporción al trabajo (el $58,6 \%$ ) y a realizar las compras (el 55,4\%). Sin embargo se aprecia que buena parte de estos hogares también se desplazan caminando o en bicicleta a realizar estas compras (el 38,9\%), y son en este caso mayoría quienes de desplazan por medios no motorizados a la escuela (el $48 \%$ de los hogares). Cabe concluir que estos hogares también buscan estrategias para economizar en sus gastos de transporte, seleccionando la proximidad para realizar ciertas actividades (compras y educación) a las que se desplazan de forma no motorizada.

TABLA 5

Medios de transporte utilizados para acudir a realizar las diferentes actividades del grupo de hogares de gasto considerable en microbús

\begin{tabular}{|c|r|r|r|r|r|}
\hline & Compras & Escuela & Trabajo & Médico & Hospital \\
\hline $\begin{array}{c}\text { Andando o } \\
\text { bicicleta }\end{array}$ & $62,5 \%$ & $37,0 \%$ & $20,6 \%$ & $28,4 \%$ & $3,0 \%$ \\
\hline Automóvil & $10,2 \%$ & $3,7 \%$ & $2,9 \%$ & $4,5 \%$ & $11,9 \%$ \\
\hline $\begin{array}{c}\text { Transporte } \\
\text { público }\end{array}$ & $27,3 \%$ & $59,3 \%$ & $76,5 \%$ & $67,0 \%$ & $85,1 \%$ \\
\hline
\end{tabular}

Fuente: Encuesta de Movilidad Municipios Sur AMG 2015

Por último los hogares que dedicaban una proporción importante de su ingreso al transporte, y preferentemente al microbús, utilizan este medio para desplazarse sobre todo a los hospitales (85,1\% de los hogares, dado la lejanía donde su ubican), al trabajo (76,5\%, intentando ampliar por encima de las inmediaciones sus búsquedas laborales), al médico de atención primaria (el $67 \%$ de todos los hogares) o a la escuela (el 59,3\% de los hogares utilizarían estos medios motorizados para ampliar su radio de acción). Para realizar las compras, sin embargo, los medios de transporte más utilizado por estos hogares son los no motorizados, suponiéndose que se ubicaría esta actividad en entornos de mayor cercanía, y que aquí también se pondrían en marchas estrategias para ahorrar en los gastos de transporte.

\subsection{LOS TIEMPOS DE TRASLADO A LAS PRINCIPALES ACTIVIDADES}

Después de la revisión bibliográfica, se optó por considerar el tiempo medio que los hogares tardaban en llegar a realizar las distintas actividades como uno de los indicadores más importantes para medir la accesibilidad urbana. Esta era una fórmula que permitía insertar los desplazamientos a los distintos lugares dentro de las dinámicas y horarios cotidianos de los hogares. Se realizó un estudio de diferencias de medias ANOVA de los tiempos empeñados en estos traslados, según el medio de transporte elegido. Todas las diferencias de medias encontradas fueron significativas. La siguiente tabla muestra los datos encontrados:

Dadas las limitaciones sobre el alcance y extensión de los medios de transporte no motorizados, quienes los utilizan empeñan entre 10 y 14 minutos de media para trasladarse a las diferentes actividades, lo que equivaldría a ubicar el radio de acción entre 1 y 2 kilómetros respecto al origen del viaje. En todos estos casos, cabe suponer que los emplazamientos se encuentran en la proximidad al origen del viaje, y que en ese pequeño radio de acción no se dispone de la oportunidad de buscar con profundidad locaciones alternativas. Dadas las limitaciones comentadas, las desviaciones sobre la media son escasas, lo que hace que el límite 
superior más desfavorable de desplazamiento se dé en el caso del desplazamiento al trabajo, por un total de 17,64 minutos.

TABLA 6

Tiempos medios en minutos de traslado a las principales actividades, según el medio de transporte elegido

\begin{tabular}{|c|r|r|r|r|r|}
\hline & Compras & Escuela & Trabajo & Médico & Hospital \\
\hline $\begin{array}{c}\text { Andando o } \\
\text { bicicleta }\end{array}$ & 10,25 & 10,05 & 14,14 & 11,56 & 12,57 \\
\hline Automóvil & 18,60 & 28,57 & 35,13 & 28,87 & 43,11 \\
\hline $\begin{array}{c}\text { Transporte } \\
\text { público }\end{array}$ & 23,28 & 46,94 & 53,91 & 37,07 & 60,01 \\
\hline Total & 15,02 & 24,18 & 40,63 & 26,01 & 52,07 \\
\hline
\end{tabular}

Fuente: Encuesta de Movilidad Municipios Sur AMG 2015

Respecto a los medios motorizados, que permiten ampliar los alcances en la búsqueda de opciones para desarrollar las diversas actividades, se aprecia la ventaja que supone el uso del automóvil frente al transporte público, fundamentalmente el microbús. Donde menos se siente esta desventaja es en el traslado a realizar las compras, puesto que el transporte público sólo penaliza un $25,16 \%$ más de tiempo respecto al acudir en automóvil. Donde más clara es la desventaja de trasladarse en microbús frente a trasladarse en automóvil es en la asistencia a las escuelas. Aquí, quienes van en el primer medio de transporte empeñan en término medio un 64,29\% más de tiempo que quienes van en automóvil.

En estas consideraciones conviene también atender a las desviaciones que se establecen respecto a los parámetros medios, que son más destacadas en el caso de quienes se desplazan en transporte público al trabajo y a los hospitales. En el primer caso, el límite superior del intervalo de confianza alrededor de la media se situaría en los 57,85 minutos invertidos, y en el caso del hospital ese intervalo se ubicaría por encima de la hora, en los 66,01 minutos.

\subsection{LAS DEMORAS EN LA ASISTENCIA A LAS DIFERENTES ACTIVIDADES}

Otro de los aspectos importantes para conocer las condiciones de accesibilidad urbana y que han sido tratados con menor detenimiento tiene que ver con las demoras en que se ha incurrido por motivos del transporte. En el diseño de la encuesta se incluyó este tipo de pregunta para comprobar si el medio de transporte utilizado incidía en esta variable. El análisis estadístico realizado indica que todas las asociaciones encontradas entre estas variables son significativas. Existe una asociación más estrecha entre el llegar tarde a la escuela y el medio de transporte utilizado ( $\mathrm{V}$ de Cramer 0,509), mientras que la asociación más leve la encontramos entre el llegar tarde al hospital y el medio utilizado (V de Cramer 0,151)

La siguiente tabla muestra el porcentaje de hogares que incurrieron en este tipo de demoras según las distintas actividades y los medios de transporte elegidos.

TABLA 7

Porcentaje de hogares que llegaron más tarde de lo previsto a realizar sus actividades por motivos del transporte, según medios de transporte utilizados

\begin{tabular}{|c|r|r|r|r|r|}
\hline & Compras & Escuela & Trabajo & Médico & Hospital \\
\hline $\begin{array}{c}\text { Andando } \\
\text { o bicicleta }\end{array}$ & $5,0 \%$ & $9,7 \%$ & $5,9 \%$ & $6,6 \%$ & $6,7 \%$ \\
\hline Automóvil & $17,4 \%$ & $44,2 \%$ & $41,4 \%$ & $26,2 \%$ & $34,2 \%$ \\
\hline $\begin{array}{c}\text { Transport } \\
\text { e público }\end{array}$ & $28,2 \%$ & $61,4 \%$ & $60,2 \%$ & $41,9 \%$ & $40,7 \%$ \\
\hline Total & $12,7 \%$ & $30,8 \%$ & $41,7 \%$ & $25,5 \%$ & $36,9 \%$ \\
\hline
\end{tabular}

Fuente: Encuesta de Movilidad Municipios Sur AMG 2015 
En primer lugar quienes consignaron un menor número de demoras para todas las actividades consideradas fueron quienes se desplazaron de manera no motorizada. La mayor proporción es en el caso de los traslados no motorizados a escuela, donde en el 9'7\% de los hogares se incurrió en al menos una demora en el último mes debido al medio de transporte. A este respecto hay que señalar que el desplazamiento no motorizado, por ubicarse en un espacio de proximidad, y por no incurrir en demasiadas contingencias derivadas del estado del medio que se transita, comporta unas altas posibilidades de previsibilidad que impiden en la mayor parte de las circunstancias llegar tarde.

Frente al uso de los medios no motorizados, los desplazamientos motorizados implican el tener que asumir una serie de contingencias derivadas del estado del medio. En el caso del automóvil, estas contingencias se resumen en los congestionamientos viales. En el caso del transporte colectivo tipo microbús, hay que añadir también las demoras en la espera de las unidades y las irregularidades en el cumplimiento de los horarios y de la prestación del servicio. Todo ello explica la gran ventaja que tienen los hogares que cuentan con automóvil, sobre los que tienen que confiar en el transporte colectivo, a la hora de evitar las demoras. En términos generales, es la escuela y el trabajo las dos actividades donde existen más hogares que señalan haberse demorado en el último mes de referencia. Resalta el caso de quienes van en transporte público, pues más del $60 \%$ de los hogares que lo utilizan para llegar a este tipo de actividades llegaron al menos una vez tarde en el último mes. Quienes acuden en automóvil no están exentos de los retardos, aunque en menor medida que quienes van en transporte público lo que implica que, incluso los hogares más aventajados que cuentan con la herramienta por excelencia de movilidad, dada la estructura territorial existente, sufren también las dificultades para acceder a los servicios urbanos.

\subsection{LA PREDISPOSICIÓN A BUSCAR OTROS EMPLAZAMIENTOS PARA LAS ACTIVIDADES}

Se introdujo también en la encuesta la pregunta sobre si se estaría dispuesto a buscar un lugar más conveniente para el desarrollo de las actividades más significativas en caso de que existieran mayores facilidades de transporte, una pregunta que resulta un buen indicador sobre el grado de satisfacción de la opción presente y cómo el transporte puede estar mermándola. Todas las asociaciones encontradas son estadísticamente significativas. La más fuerte es la que se establece entre la predisposición a buscar otro lugar más conveniente para trabajar según el medio de transporte utilizado para acudir a realizar esa actividad (V de Cramer 0,433), y la más baja entre la predisposición a buscar otro lugar más conveniente para realizar las compras y el medio elegido para realizar esta actividad (V de Cramer 0,208).

Los porcentajes particulares para cada una de las actividades son los siguientes:

TABLA 8

Porcentaje de hogares que buscarían cambiar el lugar de realización de las actividades en caso de mayores facilidades de transporte, según el medio de transporte utilizado

\begin{tabular}{|c|r|r|r|r|r|}
\hline & Compras & Escuela & Trabajo & Médico & Hospital \\
\hline $\begin{array}{c}\text { Andando o } \\
\text { bicicleta }\end{array}$ & $19,8 \%$ & $19,2 \%$ & $11,8 \%$ & $20,9 \%$ & $13,3 \%$ \\
\hline Automóvil & $22,0 \%$ & $28,9 \%$ & $31,4 \%$ & $21,4 \%$ & $32,6 \%$ \\
\hline $\begin{array}{c}\text { Transporte } \\
\text { público }\end{array}$ & $43,9 \%$ & $61,2 \%$ & $63,2 \%$ & $47,8 \%$ & $57,6 \%$ \\
\hline Total & $24,7 \%$ & $33,5 \%$ & $41,4 \%$ & $31,6 \%$ & $47,0 \%$ \\
\hline
\end{tabular}

Fuente: Encuesta de Movilidad Municipios Sur AMG 2015

Los datos son en cierta medida paradójicos y pueden suscitar interesantes hipótesis. En primer lugar llama la atención que quienes se desplazan andando o en bicicleta sean siempre quienes menos dispuestos estarían a buscar otros emplazamientos en caso de contar con 
mayores facilidades de transporte. El caso más destacado es el del trabajo. Sólo el 11,8\% de los hogares que se desplazan caminando o en bicicleta buscarían otro centro para trabajar. Posteriores investigaciones, sobre todo de naturaleza cualitativa, habrán de indagar si quienes van de manera no motorizada a trabajos en la proximidad no habrían ya renunciado a valorar el trabajo por los beneficios económicos y de trayectoria laboral, y lo valorasen por el simple hecho de que se encuentra cerca de la residencia y no exige largos y penosos desplazamientos; esto podría estar explicando la satisfacción con el lugar donde se desarrolla la actividad del trabajo. En la literatura analizada se ha puesto de manifiesto que muchos de los integrantes de estos hogares pobres pueden poner en marcha ciertos mecanismos psicológicos de autorenuncia, de manera que estarían censurando su búsqueda de locaciones alternativas para sus actividades pero que pudieran ubicarse a mayor distancia de la residencia, conformándose con las opciones más cercanas (ADEEL et al., 2016).

Quienes se trasladan en transporte motorizado podrían estar abiertos a esta exploración de opciones más lejanas y, por tanto, podrían ser más susceptibles de desear cambiar el emplazamiento de su actividad en caso de que tuvieran más facilidades de transporte. El caso más señalado es el de quienes se trasladan en transporte colectivo, fundamentalmente microbús. Dada las grandes desventajas que implica este medio de transporte, este grupo sería más sensible a buscar lugares más provechosos en espacios más distantes o más inaccesibles. Es especialmente importante el traslado al trabajo, la escuela o el hospital, donde alrededor del $60 \%$ de los hogares que viajan en microbús desearía cambiar de locación en caso de que tuvieran mayores facilidades de transporte.

Por su parte tampoco los que utilizan el automóvil están enteramente satisfechos con los lugares a donde les permite llegar. Una buena proporción buscaría lugares más convenientes en caso de tener más facilidades de transporte, sobre todo para las actividades que podemos consignar que serían estratégicas para ellos: los hospitales (un 32,6\% de los hogares que van en automóvil buscarían otro emplazamiento mejor), los trabajos (un 31,4\% de los hogares), y la escuela (un $28,9 \%$ de los hogares buscarían un mejor emplazamiento).

\subsection{CONSIDERACIONES FINALES}

Este artículo ha intentado profundizar en el conocimiento de la accesibilidad urbana de los hogares en municipios no centrales de una metrópolis ubicada en un país en desarrollo. Para hacerlo, aparte de explorar cuestiones más tradicionales como los gastos o los tiempos empeñados para acceder a los principales servicios, también se han introducido otras consideraciones sobre la apreciación de las características de ese acceso, como las demoras o la predisposición a cambiar de espacio para el desarrollo de las actividades.

Una primera y tentativa conclusión, avalada también por parte de la literatura analizada (DIAZ OLVERA et al., 2013), se refiere al amplio número de hogares que, pese a vivir en espacios no centrales y con escasa provisión de servicios urbanos, incurren en bajos gastos en transporte y se desplazan mayoritariamente caminando o en bicicleta por sus espacios más cercanos. Mientras que desde la literatura sobre el transporte acuñada en los países desarrollados, y que está buscando los mecanismos para fomentar un cambio de uso de medios no sustentables hacia otros más sustentables (HULL, 2011), este hecho merecería celebrarse, debemos sin embargo circunscribirlo en las características de los entornos urbanos propios de los países en desarrollo. En el caso de los países desarrollados, sobre todo europeos, la amplia gama de oferta de ubicaciones podría permitir un reajuste en el uso de los medios de transporte sin un menoscabo de la accesibilidad urbana. Sin embargo en el caso de las ciudades en desarrollo hay que señalar la gran carestía del medio y cómo el confiar sólo en medios no motorizados puede implicar graves dificultades para que los hogares puedan acceder a los servicios más básicos.

En estos países en desarrollo, caminar no es tanto una opción a favor de una movilidad más sustentable, sino una obligación (DIAZ OLVERA et al., 2013) que se torna empobrecedora (CAHILL, 2010) cuando se tienen que atravesar vecindarios sin infraestructuras, desolados y amenazados por la inseguridad. Además, este sentido casi obligado de la selección del caminar implica que los hogares renuncien a mejores opciones laborales, educativas o sanitarias que 
pudieran encontrarse más lejos (DIAZ OLVERA et al., 2013), e impone una ética de la autorestricción y la resignación con los escasos y precarios recursos que se cuentan en las proximidades (LAU, 2011).

Respecto a los hogares que siguen atentos a las opciones presentes en la lejanía, y se desplazan abordo de los microbuses, ya se ha visto que en muchos casos el peso del gasto de los billetes puede suponer proporciones casi prohibitivas de los ingresos familiares mensuales. El uso del transporte colectivo motorizado, en un entorno de pobreza e insuficiencia de servicios, puede significar el intento en condiciones de desventaja por obtener los mejores recursos posibles explorando opciones a media y larga distancia para desarrollar las principales actividades. Sin embargo quienes así lo hacen se demoran en largos y penosos desplazamientos.

En mejor posición respecto a la accesibilidad urbana estaría aquel grupo que se desplaza fundamentalmente en automóvil a sus distintas actividades. Dada la modestia de los ingresos en los municipios no centrales de la ciudad, sin embargo estos hogares no estarían realizando un uso indiscriminado de este medio de transporte, sino que lo emplearían para llegar a aquellas actividades estratégicas: el trabajo y los hospitales. Los usuarios del automóvil tendrían la posibilidad de ampliar su radio de búsqueda para desarrollar sus actividades más significativas, y además, según se ha visto, lo estarían haciendo en mejores condiciones respecto al tiempo o las demoras que quienes usan el microbús. Sin embargo, que buena parte de los hogares que se desplaza en automóvil señale llegar tarde a los lugares, o que indique su deseo de cambiar de emplazamiento en caso de tener mayores facilidades de transporte, muestra los graves problemas de accesibilidad que existen incluso para quienes poseen la mejor herramienta para desplazarse por un entorno disperso y sin servicios.

Estas consideraciones deben hacernos repensar ese juicio ya hecho e importado de los países desarrollados y que señala la necesidad de reorientar toda la política del transporte hacia desincentivar el uso del automóvil y favorecer medios no motorizados o el uso del transporte colectivo. En las zonas no centrales de unas metrópolis en desarrollo donde hay una gran carencia de servicios, comercios y trabajos, y donde los sistemas de transporte se resumen en la multiplicación de rutas de microbuses desorganizadas, lentas y tortuosas, esas recomendaciones importadas deben ser revisadas; en estos contextos confiar en ese transporte colectivo o en los medios no motorizados puede implicar graves problemas de acceso a los servicios más básicos para un contingente muy voluminoso de la población.

No quiero cerrar este artículo sin señalar algunas limitaciones de la investigación realizada que puedan suponer próximas líneas de estudio. En primer lugar debe de ubicarse territorialmente a cada uno de los grupos de usuarios del transporte. Todos ellos comparten el hecho de ubicarse en espacios no periféricos de la metrópoli, pero aún dentro de estas coronas existe una gran variabilidad que en su momento habrá de ser abordada y georreferenciada, con el ánimo de discriminar accesibilidades urbanas diferenciales. Además, hay que considerar que este artículo se basó en el uso que hacen los pobladores de los distintos medios de transporte para acceder a los emplazamientos básicos para su reproducción cotidiana. Queda complementar este tipo de investigación con análisis y propuestas que descansen en la propia distribución sobre el territorio de esos lugares y en mejoras en los procedimientos de planificación urbana como una forma de incidir en la mejor accesibilidad de los sujetos.

\section{REFERENCIAS}

ADEEL, M., GAR-ON YEH, A. y ZHANG, F. (2016): Transport Policy. "Transportation Disadvantage and Activity Participation in the Rawalpindi and Islamabad, Pakistan", no 47, p. 1-12.

AOUN, N., MATSUDA, H. y SEKIYAMA, M. (2015): Social Science \& Medicine. "Geographical Accessibility to Healthcare and Malnutrition in Rwanda", n' 130, p. 135-145.

BEJLERI, I., STEINER, R. L., FISCHMAN, A. y SCHUMKER, J. (2011): Urban Design International. "Using GIS to Analyze the Role of Barriers and Facilitators to Walking in Children's Travel to School", vol. 16, n 1, p. 51-62. 
BANISTER, D. Y LICHFIELD, N. (1995): Transport and Urban Development. "The Key Issues in Transport and Urban Development". En Banister D. y Lichfield, N. (eds.). E \&FN Spon, London, p.1-17

BANISTER, D. (2002): Transport Planning. Spon Press, New York, 317 p.

BATTY, M. (2009): International Encyclopedia or Human Geography. "Urban Modeling". KITCHIN, R. Y THRIFT, N. (eds.). Elsevier, London, p. 51-58

BHAT, C. R, GOULIAS, K. G, PENDYALA, R.M, PALETI, R., SIDHARTHAN, R., SCHMITT, L. y HU, H. H. (2013): Transportation. "A Household Level Activity Pattern Generation Model with Application for Southern California", n 40, p. 1063-1086.

BIBA, G., DES ROSIERS, F., THERIAULT, M. y VILLENEUVE, P. (2006): Journal of Real Estate Literature. 'Bix Boxes versos Traditional Shopping Centers: Looking at Households' Shopping Trip Patterns", vol. 14, no 2, p. 175-202.

BLANFORD, J. I., KUMAR, S., LUO, W. y MACEACHREN, A. M (2012): International Journal of Health Geographics. "It's a Long, Long Walk: Accessibility to Hospitals, Maternity and Integrated Health Centers in Niger", vol. 11, no 24, p. 1-24.

BRINDLE, R. (2003): Road \& Transportation Research. "Kicking the Habit. Some Musings on the Meaning of 'Car Dependence'", , vol. 12, n 3, p. 61-73.

CASTILLO-MANZANO, J. I. y LÓPEZ VALPUESTA, L. (2009): Cities. "Urban Retail Fabric and the Metro: A Complex Relation. Lessons from Middle-Sized Spanish Cities", n ${ }^{\circ}$ 26, p. 141-147.

CAHILL, M. (2010): Transport, Environment and Society. Open University Press, Maidenhead, $162 \mathrm{p}$.

CLIFTON, K. y LUCAS, K. (2004): Running on Empty. Transport, Social Exclusion and Environmental Justice. "Examining the Empirical Evidence of Transport Inequality in the US and UK". En Lucas, K. (ed.). Policy Press, Bristol, p. 15-38

COVENEY, J. y O'DWYER, L. (2009): Health \& Place. "Effects of Mobility and Location in Foods Access", no 15, p. 45-55.

DELGADO, M. E. M. y CANTERS, F. (2011): Singapore Journal of Tropical Geography. "Measuring the Accessibility of Different Household Income Groups to Basic Community Services in Upland Misamis Oriental, Northern Mindanao, Philippines", n 32, p. 168-184.

DIAZ OLVERA, L., PLAT, D. y POCHET, P. (2003): Transport Policy. "Transportation Conditions and Access to Services in a Context of Urban Sprawl and Deregulation. The Case of Dar el Salaam", n 10, p. 287-298.

DIAZ OLVERA, L., PLAT, D. y POCHET, P. (2013): Journal of Transport Geography. "The Puzzle of Mobility and Access to the City in Sub-Saharan Africa", n' ${ }^{\circ} 32$, p. 56-64.

DONG, X., BEN-AKIVA, M. E, BOWMAN, J. L. y WALKER J. L. (2006): Transportation Research Part A. "Moving from Trip-Based to Activity-Based Measures of Accessibility", $\mathrm{n}^{\circ}$ 40, p. 163-180.

ETTEMA, D. y TIMMERMANS, H. (2007): Geographical Analysis. "Space-Time Accessibility Under Conditions of Uncertain Travel Times: Theory and Numerical Simulations", no 39, p. 217-240.

FARBER, S., PÁEZ, A., MERCADO, R. C., ROORDA, M. y MORENCY, C. (2011): Transportation. "A Time-Use Investigation of Shopping Participation in Three Canadian Cities: Is There Evidence of Social Exclusion?", no 38, p. 17-44.

FARBER, S., MORANG, M. Z y WILDENER, M. J. (2014): Applied Geography. "Temporal Variability in Transit-Based Accessibility to Supermarkets", no 53, p.149-159.

FAULKNER, G. EJ., RICHICHI, V., BULIUNG, R. N., FUSCO, C. y MOOLA, F. (2010): Journal of Behavioral Nutrition and Physical Activity. "What's Quickest and Easiest? Parental Decision Making About School Trip Mode. International", vol. 7, nº 62, p. 1-11.

FITZPATRICK, K., HORTON, A. y PARISI, D. (2012): Institute of Transportation Engineers Journal. "Transportation Practitioners. Stakeholders in Safer Routes to School", vol. 82, nº 8, p. 24-27.

HERNÁNDEZ, D. (2012): EURE. "Activos y estructuras de oportunidades de movilidad. Una propuesta analítica para el estudio de la accesibilidad por transporte público, el bienestar y la equidad", vol. 38, n 115, p. 117-135. 
USOS DE LOS MEDIOS DE TRANSPORTE...

HERNÁNDEZ, D. y ROSSEL, C. (2015): Journal of Transport Geography. "Inequality and Access to Social Services in Latin America: Space Time Constrains of Child Care Checkups and Prenatal Care in Motevideo", n 44, p. 24-32.

HEYNEN, N. (2006): In the Nature of Cities. Urban Political Ecology and the Politics of Urban Metabolism. "Justice of Eating in the City: The Political Ecology of Urban Hunger". En Heynen, N., Kaika, M. y Swyngedouw, E. (eds.). Routledge, London, p. 124-136.

HORNER, M. W. y WOOD, B. S. (2014): Applied Geography. "Capturing Individual's Food Environments Using Flexible Space-Time Accessibility Meassures", no 51, p. 99-107.

HORNER, M. W., DUNCAN, M. D., WOOD, B. S., VALDEZ-TORRES, J. y STANSBURY, C. (2015): Travel Behavior and Society. "Do Aging Populations Have Differential Accessibility to Activities? Analyzing the Spatial Structure of Social, Professional, and Business Oportunities", n 2, p. 182-191.

HULL, A. (2011): Transport Matters. Integrated Approaches to Planning City-Regions. Routledge, London, $295 \mathrm{pp}$.

JIRÓN, P. (2004): The Beginning of the End of the Chilean Housing Model: Lessons to be Learned from over 20 Years of Experience. International Conference Adequate \& Affordable Housing for All, Toronto, $15 \mathrm{pp}$.

KELLERMAN, A. (2006): Personal Mobilities. Routledge, London, 212 pp.

KENYON, S. (2011): Journal of Transport Geography. "Transport and Social Exclusion: Access to Higher Education in the UK Policy Context", no 19, p. 763-771.

KIM, S. y ULFARSSON, G. F. (2008): Transportation. "Curbing Automobile Use for Sustainable Transportation: Analysis of Mode Choice on Short Home-Based Trips", $\mathrm{n}^{\circ}$ 35, p. 723-737.

LAU, J. C.-Y. (2011): Cities. "Spatial Mismatch and the Affordability of Public Transport for the Poor in Singapore's New Towns", n 38, p. 230-237.

LAU, J. C.-Y y CHIU, C.C.H. (2003): Cities. "Accessibility in Low-Income Workers in Hong Kong" vol. 20, nº 3, p. 197-204.

LE VINE, S., LEE-GOSSELIN, M., SIVAKUMAR, A. y POLAK, J. (2013): Journal of Transport Geography. "A New Concept of Accessibility to Personal Activities. Development of Theory and Application to an Empirical Study of Mobility Resource Holdings", no 31, p. 1-10.

LEWIS, P. G y BALDASSARE, M. (2010): Journal of the American Planning Association. "The Complexity of Public Attitudes Toward Compact Development", vol. 76, nº 2, p. 219237.

LINDÓN VILLORIA, A. (1997): De la expansión urbana y la periferia metropolitana. El Colegio Mexiquense, Zinacantepec, $22 \mathrm{pp}$.

LUCAS, K. (2004): Running on Empty. Transport, Social Exclusion and Environmental Justice. "Locating Transport as a Social Policy Problem". En Lucas, K. (ed.). Policy Press, Bristol, p. 7-14.

MANDERSCHEID, K. (2009): Mobilities and Inequality. "Unequal Mobilities". En Ohnmacht, T., Maksim, H. y Bergman, M. M. (eds.). Aldershot, Farnham, p. 27-51.

MARQUET, O. y MIRALLES-GUASCH, C. (2014): Transportation Research Part A. "Walking Short Distances: The Socioeconomic Drivers for the Use of Proximity in Everyday Mobility in Barcelona", $\mathrm{n}^{\circ}$ 70, p. 210-222.

MARQUET, O. y MIRALLES-GUASCH, C. (2014b): Cities. "The Walkable City and the Importance of the Proximity Environments for Barcelona's Everyday Mobility", vol. 42, p. 258-266.

MASOUMI, H. E. (2014): Management Research and Practice. "Urban Sprawl in Mid-Sized Cities of Mena. Evidence from Yazd and Kashan in Central Iran", vol. 6, no 2, p. 25-41.

MCDONALD, N. C., STEINER, R., CHANAM, L., SMITH, T. R., ZHU, X. y YAN, Y. (2014): Journal of the American Planning Association. "Impact of the Safe Routes to School Program on Walking and Bicycling", vol. 80, $\mathrm{n}^{\circ}$ 2, p. 153-167.

MOAVENZADEH, F. y MARKOW, M J (2007): Moving Millions. Transport Strategies for Sustainable Development in Megacities. Springer, Dordrecht, 269 pp. 
NEUTENS, T. (2015): Journal of Transport Geography. "Accesibility, Equity and Health Care. Review and Research Directions for Transport Geographers", no 45, p. 14-27.

NEWMAN, M. (2009): Transit Oriented Development. "Planning for Transit Oriented Development: Strategic Principles". CURTIS, C., RENNE, J.L. y BERTOLINI, L. (Eds.). Ashgate, Farnham, p. 13-22.

OHNMACHT, T., MAKSIM, H. y BERGMAN, M. M. (2009): Mobilities and Inequality. "Mobilities and Inequalities. Making the Connections". En Ohnmacht, T., Maksim, H. y Bergman, M. M. (eds.). Aldershot, Farnham, p. 7-26.

PINJARI, A. R., PENDYALA, R., BHAT, C. R. y WADDELL, P. A. (2011): Transportation. "Modeling the Choice Continuum. An Integrated Model of Residential Location, Auto Ownership, Bicycle Ownership, and Commute Tour Mode Choice Decisions", vol. 38, p. 933-958.

PLAUT, P. (2004): Transportation. "Non-Commuters: The People Who Walk to Work or Work at Home", vol. 31, no 2, p. 229-255.

REIMERS, V. (2013): Transportation Research Part A. "Convenience for the Car-Borne Shopper: Are Malls and Shopping Strips Driving Customers Away?", n 49, p. 35-47.

RODRÍGUEZ VIGNOLI, J. (2008): EURE. "Movilidad cotidiana, desigualdad social y segregación residencial en cuatro metrópolis de América Latina", vol. 34, no 103, p. 49-71.

SABATINI, F. y BRAIN I. (2008): EURE. "La segregación, los guetos y la integración social urbana: mitos y claves", vol. 34, no 103, p. 5-26.

SALZE, P., BANOS, A., OPPERT, J.-M., CHARREIRE, H., CASEY, R., SIMON, C., CHAIX, B., BADARIOTTI, D. y WEBER, C. (2011): International Journal of Health Geographics. "Estimating Spatial Accesibility to Facilities on the Regional Scale: An Extended Commuting-Based Interaction Potential Model", vol. 10, nº 2, p. 1-16.

SEO, S.-E., OHOMORI, N. y HARATA, N. (2013): Transportation. "Effects of Household Structure and Accesibility on Travel", $\mathrm{n}^{\circ}$ 40, p. 487-465.

SERULLE, N.U. y CIRILLO, C. (2016): Public Transportation. "Transportation Needs of Low Income Population: A Policy Analysis for the Washington DC Metropolitan Region", no 8, p. 103-123.

SIMMONDS, D. y ECHENIQUE, M. (1999): Review of Land-Use/Transport Interaction Models. Department of the Environment, Transport and Regions, London, $72 \mathrm{p}$.

SOCIAL EXCLUSION UNIT (2003): Making the Connections. Final Report on Transport and Social Exclusion. Office of the Deputy Prime Minister, London, $147 \mathrm{pp}$.

SRINIVASAN, S. y ROGERS, P. (2005): Journal of Transport Geography. "Travel Behaviour of Low-Income Residents: Studying Two Contrasting Locations in the City of Chennai, India", nº 13, p. 267-274.

SUSILO, Y. O y AXHAUSEN, K. W. (2014): Transportation. "Repetitions in Individual Daily Activity-Travel-Location Patterns: A Study Using the Herfindahl-Hirschman Index", no 41, p. 995-1011.

SWEET, M. N y CHEN, M. (2011): Transportation. "Does Regional Travel Time Unreliability Influence Mode Choice?, no 38, p. 625-642.

URRY, J., (2006): Against Automobility. "Inhabiting the Car". En Bohm, S.(ed.). Oxford, Blackwell, p. 17-31.

WANG, C.-H. y CHEN, N. (2015): Transportation. "A GIS Based Spatial Stadistical Approach to Modeling Job Accessibility by Transportation Mode: A Case Study of Columbus, Ohio", $\mathrm{n}^{\circ} 45$, p. $1-11$.

WANG, H., LING, F., TAO, Q. y WEI, L. (2016): Applied Geography. "The Role of SocioEconomic Status and Spatial Effects on Fresh Food Access: Two Cases Studio y Canada", ${ }^{\circ}$ 67, p. 27-38.

WEGENER, M. (2008): Handbook of Transport Geography and Spatial Systems. "Overview of Land-Use Transport Models". HENSER, D.A., BUTTON, K.J., HAYNES, K.E, STOPHER, P.R. (eds.). Emerald, Bingley, p. 127-146.

WIDENER, M. J., FARBER, S., TIJS, N. y HORNER, M. W. (2013): Heath \& Place. "Using Urban Commuting Data to Calculate a Spatiotemporal Accessibility Measure for Food Environment Studies", no 21, p. 1-9. 
USOS DE LOS MEDIOS DE TRANSPORTE...

WIDENER, M. J, FARBER, S., TIJS, N. y HORNER, M. (2015): Journal of Transport Geography. "Spatiotemporal Accesibility to Supermarkets Using Public Transit: An Interaction Potential Approach in Cincinnati, Ohio", n ${ }^{\circ}$ 42, p. 72-83.

YANG, S., ZARR, R., KASS-HOUT, T., KOURSOH, A. y KELLY, N. (2006): Journal of Health Care for the Poor and the Underserved. "Transportation Barriers to Accessing Health Care for Urban Children", vol. 17, n 4, p. 928-943.

ZOLNIK, E. J. (2012): Journal of Transport Geography. "The Cost of Sprawl for Private Vehicle Commuters", nº 20, p. 23-30. 